\title{
Buffer Block Planning for Interconnect Planning and Prediction *
}

\author{
Jason Cong, Tianming Kong and David Zhigang $\operatorname{Pan}^{\dagger}$ \\ Department of Computer Science, University of California, Los Angeles, CA 90095 \\ ${ }^{\dagger}$ IBM T. J. Watson Research Center, Yorktown Heights, NY, 10598
}

\begin{abstract}
This paper studies buffer block planning for interconnect planning and prediction in deep submicron designs. We first introduce the concept of feasible region for buffer insertion, and derive its closedform formula. We observe that the feasible region for a buffer is quite large in general even under fairly tight delay constraint. Therefore, FR gives us a lot of flexibility to plan for buffer locations. We then develop an effective buffer block planning algorithm to perform buffer clustering such that design objectives such as overall chip area and the number of buffer blocks can be minimized. Effective buffer block planning can plan and predict system-level interconnect by construction, so that accurate interconnect information can be used in early design stages to ensure design closure.
\end{abstract}

\section{Introduction}

For deep submicron (DSM) VLSI designs, it is well known that interconnect has become the dominant factor in determining the overall circuit performance and complexity. To improve the interconnect performance, many interconnect optimization techniques have been proposed recently, such as topology construction, driver sizing, buffer insertion, wire sizing and spacing (see [1] for a tutorial). Among them, buffer insertion in particular, is a very effective and useful technique by inserting active devices (buffers) to break original long interconnects into shorter ones such that the overall delay can be reduced. It has been shown that without buffer insertion, the interconnect delay for a wire increases about quadratically as wire length increases, but it only increases linearly under proper buffer insertion [2, 3, 4]. As an example, [5] showed that the delay of a $2 \mathrm{~cm}$ global interconnect can be reduced in a factor of $7 \mathrm{x}$ by optimal buffer insertion. As the intrinsic delay of a buffer (i.e., the cost for buffer insertion) becomes smaller and the chip dimension gets larger, it is expected that a large number of buffers shall be inserted for high-performance designs in current and future technology generations (e.g., close to 800,000 for $50 \mathrm{~nm}$ technology as estimated in [6]). The introduction of so many buffers will significantly change a floorplan and wire length distribution. Thus they shall be planned as early as possible. By embedding such a buffer planning process into physical design flow (e.g., during floorplanning), one can estimate the interconnect parameters (length, timing, and so on) accurately for each individual net, so that timing closure and design convergence may be better achieved.

However, most existing buffer insertion algorithms (e.g., [7, 8, 9, 10]) were designed for post-layout interconnect optimization, and for a single net only. There was no global planning for tens of thousands of nets that may need buffer insertion to meet their performance requirement as in DSM designs. Meanwhile, most existing floorplanning algorithms (e.g., [11, 12, 13]) only focused on wirelength/area minimization, and did not consider buffer insertion for performance optimization. [14] considered buffer insertion during floorplanning, but it simply assumed that buffers can be inserted anywhere in an existing floorplan. However, not as wires which may have over-the-cell routing structure (given multiple metal layers), buffers must consume silicon resource and require connections

*This research is partially sponsored by Semiconductor Research Corporation under Contract 98-DJ-605, and equipment donation from Intel. to power/ground network, thus may not be placed anywhere (e.g., no inside some hard IP blocks). Otherwise, it will seriously impact the hierarchical design style, make it difficult to use/reuse IP blocks. As a result, the designers often prefer to form buffer blocks between existing circuit blocks of current floorplan. If there is no careful planning of these large amount of buffers, one may get excessive area increase. Moreover, without careful planning, it is most likely that these buffers will be distributed rather randomly over the entire chip, which will definitely complicate global/detailed routing and power/ground distribution.

To effectively address the above issues, as part of our general effort of developing an interconnect-centric design flow [15], we study in this paper the buffer block planning (BBP) problem, which automatically generates buffer blocks for interconnect optimization during physical-level floorplanning. It considers buffer location constraints (e.g., hard IP blocks and pre-design layout), and provides more regular buffering structure for layout and power/ground networks. Since the buffer block planning roughly determines every buffer location for each net, we can then obtain more accurate wire length and congestion estimation and prediction during physical design.

Our major contributions of this paper include:

- We first introduce the concept of feasible region (FR) for buffer insertion under certain delay constraint, and derive an analytical formula for it.

- We find that the FR for a buffer can be surprisingly large, even under tight delay constraint. This crucial observation provides us a lot of flexibility to plan a buffer's location.

- We propose to use buffer blocks to appropriately cluster individual buffers together so that the total chip area due to buffer insertion, as well as the number of buffer blocks can be minimized.

- We develop an effective algorithm for buffer block planning. It can be used as a key element for interconnect-driven floorplanning, on-line interconnect estimation and prediction.

To the best of our knowledge, this is the first in-depth study of buffer block planning. The rest of the paper is organized as follows. Section 2 formulates the problem. Section 3 derives the feasible region for buffer insertion. Section 4 studies buffer block planning and proposes an effective algorithm for it. Experimental results are shown in Section 5, followed by the conclusion in Section 6.

\section{Problem Formulation}

The problem of buffer block planning (BBP) is formulated as follows. Given an initial floorplan and the performance constraints for each net, we want to determine the optimal locations and dimensions of buffer blocks (i.e., the extra blocks between existing circuit blocks of current floorplan) such that the overall chip area and the number of buffer blocks after buffer insertion are minimized while the performance constraint for each net is satisfied (if it is a valid timing constraint for the given floorplan that can be met by optimal buffer insertion). The output from our buffer block planning 
consists of the following information: the number of buffer blocks, each buffer block's area, location, and corresponding nets that use some buffer in this buffer block to meet the delay constraints. In this study, we focus on two-pin nets and derive the closed-form formula of feasible region for buffer insertion. The concepts of feasible region and buffer block planning can be extended to multiple-pin nets as well.

We model a driver/buffer as a switch-level RC circuit [5], and use the well-known Elmore delay model for delay computation. The key parameters for interconnect and buffer in our study are listed in Table 1 . The values are based on a $0.18 \mu \mathrm{m}$ technology in NTRS'97[16].

Table 1: Key parameters

\begin{tabular}{|l|l|r|}
\hline$r$ & unit length wire resistance $(\Omega / \mu m)$ & 0.075 \\
$c$ & unit length wire capacitance $(f F / \mu m)$ & 0.118 \\
$T_{b}$ & intrinsic delay for buffer $(p s)$ & 36.4 \\
$C_{b}$ & input capacitance of buffer $(f F)$ & 23.4 \\
$R_{b}$ & output resistance of buffer $(\Omega)$ & 180 \\
\hline
\end{tabular}

\section{Feasible Region for Buffer Insertion}

The feasible region (FR) for a buffer $B$ is defined to be the maximum region where $B$ can be located such that by inserting buffer $B$ into any location in that region, the delay constraint can be satisfied. Figure 1 illustrates the concept of FR for inserting 1 or $k$ buffers into a net where the source and sink of the net are connected by a given route. In the figure, the FR's are the shaded line segments.

\subsection{Feasible Region for Single-Buffer Insertion}

For single-buffer insertion in Figure 1(a), let us denote $x$ to be the distance from driver to buffer. We have the following theorem for its feasible region:

Theorem 1 For a given delay constraint $T_{r e q}$, the feasible region $\left[x_{\min }, x_{\max }\right]$ for inserting one buffer is

$$
\begin{aligned}
& x_{\min }=\operatorname{MAX}\left(0, \frac{K_{2}-\sqrt{K_{2}^{2}-4 K_{1} K_{3}}}{2 K_{1}}\right) \\
& x_{\max }=\operatorname{MIN}\left(l, \frac{K_{2}+\sqrt{K_{2}^{2}-4 K_{1} K_{3}}}{2 K_{1}}\right)
\end{aligned}
$$

where

$$
\begin{aligned}
& K_{1}=r c \\
& K_{2}=\left(R_{b}-R_{d}\right) c+r\left(C_{L}-C_{b}\right)+r c l \\
& K_{3}=R_{d} C_{b}+T_{b}+R_{b}\left(C_{L}+c l\right)+\frac{1}{2} r c l^{2}+r l C_{L}-T_{r e q}
\end{aligned}
$$

Proof sketch: For a single buffer insertion at distance $x$ from the driver, the total Elmore delay from the driver to the sink is

$$
\begin{aligned}
T_{1}= & R_{d}\left(c x+C_{b}\right)+\frac{1}{2} r c x^{2}+r x C_{b}+T_{b} \\
& +R_{b}\left[c(l-x)+C_{L}\right]+\frac{1}{2} r c(l-x)^{2}+r(l-x) C_{L} \\
\equiv & K_{1} x^{2}-K_{2} x+\left(K_{3}+T_{r e q}\right) \\
\leq & T_{r e q}
\end{aligned}
$$

Solving the quadratic inequality with boundary conditions, we prove the theorem.

Note that for Theorem 1 to be valid, $K_{2}^{2}-4 K_{1} K_{3} \geq 0$ shall hold. Otherwise, no feasible region exists and the initial floorplanning/timing budget has to be modified. Figure 2 shows the FR for inserting one buffer to an interconnect of length from $6 \mathrm{~mm}$ to $9 \mathrm{~mm}$. We first compute the best delay $T_{b e s t}$ by inserting one buffer, then assign the delay constraint to be $(1+\delta) T_{\text {best }}$, with $\delta$ to be from 0 to $50 \%$. The $\mathrm{x}$-axis shows the $\delta$ and the y-axis shows the FR distance, i.e., $x_{\max }-x_{\min }$. It is interesting to see that even with fairly small amount of slack, say $10 \%$ more delay from $T_{\text {best }}$, the FR can be as much as $50 \%$ of the wire length. This important observation leads great flexibility for buffer planning, to be discussed later on in this paper.

(a)

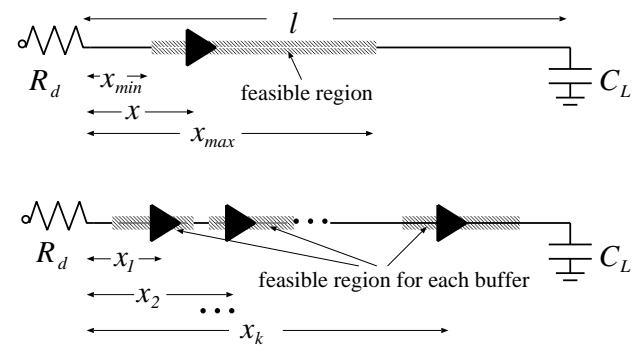

Figure 1: Feasible regions for inserting (a) one buffer; (b) $k$ buffers.

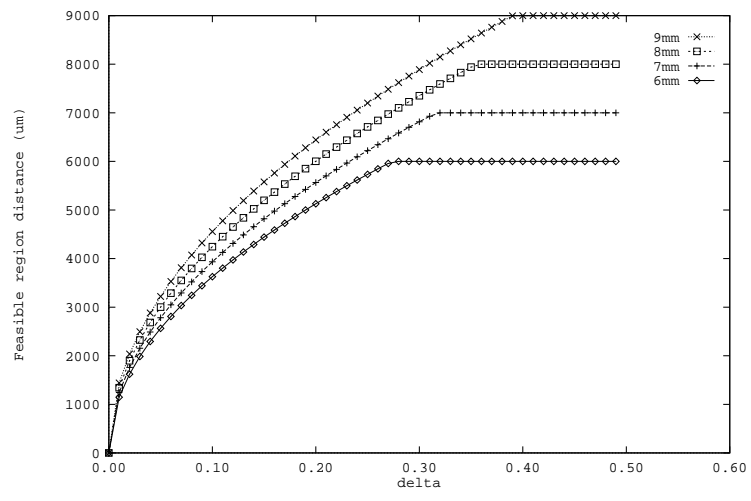

Figure 2: The distance feasible region for inserting a buffer under different delay constraint of $\delta$.

\subsection{Feasible Regions for Multiple-Buffer Insertion}

For a long interconnect, more than one buffer may be needed to meet a given delay budget. For $k$ buffers inserted, we have the following theorem to compute the feasible region for each buffer.

Theorem 2 For a long interconnect with $k$ buffers inserted, the feasible region for the $i$-th buffer $(i \leq k)$ is $x_{i} \in$ $\left[x_{\min }(k, i), x_{\max }(k, i)\right]$ with

$$
\begin{aligned}
& x_{\text {min }}(k, i)=M A X\left(0, \frac{K_{2}^{\prime}-\sqrt{K_{2}^{\prime 2}-4 K_{1}^{\prime} K_{3}^{\prime}}}{2 K_{1}^{\prime}}\right) \\
& x_{\text {max }}(k, i)=M I N\left(l, \frac{K_{2}^{\prime}+\sqrt{K_{2}^{\prime 2}-4 K_{1}^{\prime} K_{3}^{\prime}}}{2 K_{1}^{\prime}}\right)
\end{aligned}
$$


where $K_{1}^{\prime}, K_{2}^{\prime}$ and $K_{3}^{\prime}$ are functions of $k$ and $i$ (for simplicity of notation, we drop them in the above equations) with

$$
\begin{aligned}
K_{1}^{\prime}(k, i) & =\frac{(k+1) r c}{2 i(k-i+1)} \\
K_{2}^{\prime}(k, i) & =\frac{\left(R_{b}-R_{d}\right) c}{i}+\frac{r\left(C_{L}-C_{b}\right)+r c l}{k-i+1} \\
K_{3}^{\prime}(k, i) & =k T_{b}-T_{r e q}+\left[R_{d}+(i-1) R_{b}+\frac{(k-i) r l}{k-i+1}\right] \cdot C_{b} \\
& +R_{b}\left[(k-1) C_{b}+C_{L}+c l\right]+\frac{r c l^{2}}{2(k-i+1)}+r l C_{L} \\
& -\frac{(i-1) c\left(R_{b}-R_{d}\right)^{2}}{2 i r}-\frac{(k-i) r\left(C_{b}-C_{L}\right)^{2}}{2(k-i+1) c} .
\end{aligned}
$$

Proof sketch: The proof is similar to that of Theorem 1. The main difference is that there is no other buffer before or after the singlebuffer scenario, but now for the $i$-th buffer, there are $i-1$ buffers before it and $k-i$ buffers after it. Since we want to compute the maximum region that the $i$-th buffer can be placed, all other buffers may be optimally placed to minimize the delay during the computation of the feasible region of the $i$-th buffer. We use the analytical formula (6) in [17] to compute the optimal delay with multiple buffers, which is also a quadratic of $x$ (the distance of the $i$-th buffer from the driver). Then, solving a similar quadratic inequality like that in the proof of Theorem 1, we can get Theorem 2.

It can be verified that Theorem 1 is a special case of Theorem 2 with $k=i=1$. The following theorem determines the minimum number of buffers that are required to meet a given delay budget.

Theorem 3 The minimum number of buffers to meet the delay constraint $T_{r e q}$ for an interconnect of length $l$ is

$$
k_{m i n}=\left\lceil\frac{K_{5}-\sqrt{K_{5}^{2}-4 K_{4} K_{6}}}{2 K_{4}}\right\rceil
$$

where

$$
\begin{aligned}
K_{4}= & R_{b} C_{b}+T_{b} \\
K_{5}= & T_{r e q}+\frac{r}{c}\left(C_{b}-C_{L}\right)^{2}+\frac{c}{r}\left(R_{b}-R_{d}\right)^{2} \\
& -\left(r C_{b}+c R_{b}\right) l-T_{b}-R_{d} C_{b}-R_{b} C_{L} \\
K_{6}= & \frac{1}{2} r c l^{2}+\left(r C_{L}+c R_{d}\right) l-T_{r e q}
\end{aligned}
$$

Proof sketch: From (6) in [17], we know that the optimal delay for inserting $k$ buffers into a wire of length $l$ is

$$
\begin{aligned}
T_{k}= & \frac{r l\left(k C_{b}+C_{L}\right)+c l\left(R_{d}+k C_{b}\right)}{k+1} \\
& +\frac{\left(k C_{b}+C_{L}\right)\left(k R_{b}+R_{d}\right)}{k+1}+k T_{b} \\
& +\frac{r c l^{2}+\frac{k r\left(C_{b}-C_{L}\right)^{2}}{c}-\frac{k c\left(R_{b}-R_{d}\right)^{2}}{r}}{2(k+1)} .
\end{aligned}
$$

Let $T_{k}<T_{r e q}$, and write it as a quadratic inequality of $k$, we can obtain $K_{4} \cdot k^{2}-K_{5} \cdot k+K_{6}<0$, and thus Theorem 3 .

Based on these results, given a two-pin net with a delay constraint $T_{r e q}$, the required buffer number $k_{m i n}$ and the feasible region for each buffer can be computed in constant time. As a example, for a $1 \mathrm{~cm}$ net with $R_{d}=R_{b}, C_{L}=C_{b}$ and the delay constraint $T_{r e q}=1.05 \cdot T_{\text {best }}\left(T_{\text {best }}\right.$ is the best delay by optimal buffer insertion, which is $464 \mathrm{ps}$ ), we can calculate that the minimum number of buffers needed is $k_{\min }=2$, and the feasible regions for the first and second buffers are $[1.47 \mathrm{~mm}, 5.20 \mathrm{~mm}]$ and $[4.80 \mathrm{~mm}, 8.53 \mathrm{~mm}]$, respectively. Note that the FR's of adjacent buffers may overlap, as in this example. This is because FR for each buffer is computed independently, assuming all other buffers can be optimally placed to satisfy the delay constraint, i.e., our FR provides the maximum freedom for each a buffer. It shall be noticed that during the buffer planning phase (in Section 4), when a buffer is placed (i.e., "committed") to a position within its feasible region, we will need to update the FR's of all other unplaced buffers of the same net to safely meet its delay constraint. But since we have the analytical formula, this update can be computed in constant time.

\subsection{2-Dimensional Feasible Region}

So far, our discussion of FR is restricted to a one-dimensional line, i.e., we assume the route from source to sink is already specified by some global router. Thus the feasible region is also onedimensional. In practice, however, global routing usually has not been performed prior to or during floorplanning. In this case we can compute a much larger 2-dimensional FR for each buffer. This 2-dimensional feasible region is essentially the union of the onedimensional feasible regions of all possible routes from source to sink. Therefore, we can have much more freedom for buffer planning. Since for each net, its buffer location will then determine roughly its routing, our buffer block planning indeed determines the overall global routing structure for each net.

For a two-dimensional net, let the source location be $\left(x_{s r c}, y_{s r c}\right)$ and the sink location be $\left(x_{\sin k}, y_{\operatorname{sink}}\right)$. We only need to consider non-degenerate two-dimensional cases here, i.e., $x_{s r c} \neq x_{s i n k}$ and $y_{s r c} \neq y_{s i n k}$. Also, we consider only the monotone (i.e., nondetour) routes from source to sink. We prove that with Manhattan monotone routing, the 2-D FR can be obtained by the following theorem.

Theorem 4 For a net with $k$ buffers, the 2-dimensional feasible region for the $i$-th buffer is the region bounded by two parallel lines with Manhattan distances from the source to be $x_{m i n}(k, i)$ and $x_{\max }(k, i)$, respectively (the same as Theorem 2$)$, and by the rectangular bounding box between the source and the sink. The slope of the two parallel lines is either +1 or -1 , depending on the sign of $\frac{y_{s i n k}-y_{s r c}}{x_{\sin k}-x_{s r c}}$ : if $\frac{y_{s i n k}-y_{s r c}}{x_{\sin k}-x_{s r c}}>0$, the slope is -1 ; if $\frac{y_{s i n k}-y_{s r c}}{x_{\sin k}-x_{s r c}}<0$, the slope is +1 .

Proof sketch: It can be easily verified from the definition of Manhattan distance and monotone routing.

Note that in previous works of buffer insertion, buffers are mostly inserted in their delay-minimal positions, which we call them as restricted positions because they are only a small subset within our FR. The restricted positions for a 2-dimensional net can be obtained by the following corollary:

Corollary 1 For a 2-D net with $k$ buffers, the restricted positions of the $i$-th buffer for all monotone routes from the source to the sink form a restricted line within the feasible region of the $i$-th buffer. The line slope is again either +1 or -1 , the same as that in Theorem 4.

Also, if there are obstacles (such as hard IP blocks), we just need to deduct them from the feasible region. An example of a twodimensional feasible region with a restricted line and some obstacles is illustrated in Figure 3. 


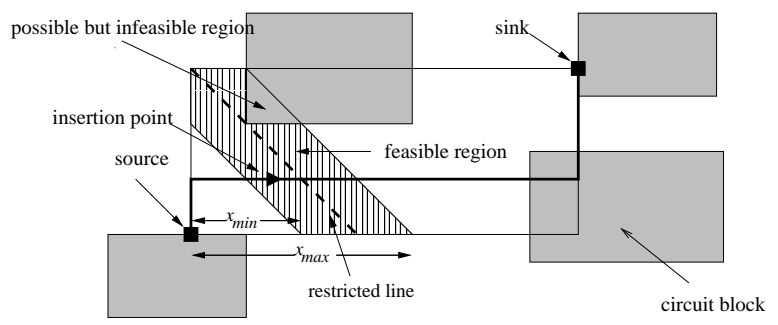

Figure 3: 2-D feasible region and a restricted line. The existing circuit blocks act as obstacles for buffer insertion.

\begin{tabular}{||l||}
\hline \hline Algorithm: Buffer Block Planning (BBP) \\
\hline 1. Build horizontal and vertical polar graph; \\
2. Build tile data structure; \\
3. For each tile, compute its area slacks; \\
4. while (there exists buffer to be inserted) \{ \\
5. tile $\leftarrow$ Pick_A_Tile(); \\
6. Insert_Buffers(tile); \\
7. $\quad$ update $W_{c}, H_{c}$, FR and area slacks; \\
8\}$. \\
\hline \hline
\end{tabular}

Figure 4: Overall flow of the buffer block planning algorithm.

\section{Buffer Block Planning}

In the previous section, we show that for a given delay constraint, a buffer may be inserted in a fairly wide feasible region. Therefore, it gives us a lot of flexibility to plan for every buffer's insertion position (within its FR) such that the overall chip area due to buffer insertion, as well as the total number of buffer blocks can be minimized. It shall be noted that such a buffer block planning also determines the overall global routing structures for long interconnects by determining their internal buffer locations. In this section, we will present an effective algorithm for buffer block planning.

The BBP problem is very difficult in the following senses: (i) Many buffer blocks might need to be optimally shaped for overall chip area minimization; (ii) To make the situation even more complicated, different buffers of the same net will not be independent of each other. For a long interconnect with more than one buffers inserted, Theorem 2 gives the maximum FR for each buffer. However, when a buffer is committed to a certain location within its FR, the FR's for other buffers in the same net will have to be updated so that the delay constraint can be safely met ${ }^{1}$.

In the rest of this section, we will present an effective algorithm to solve the buffer block planning problem. There are several important features in our BBP algorithm: (i) It takes advantage of both the flexibility of FR and the simplicity of its analytical formulae, so that one may handle large circuits with tens of thousands long interconnects easily; (ii) Since in most floorplans, there are some dead areas that cannot be taken by any circuit module, our algorithm will use these dead areas as much as possible to save the overall chip area; (iii) Different from previous buffer insertion algorithm which only inserts one buffer for a single net, our BBP algorithm always maintains global buffer insertion information for all nets, thus it can effectively cluster individual buffers that belong to different nets into buffer blocks.

Figure 4 gives the overall flow of the buffer block planning algorithm. Lines 1 to 3 are the data preparation stages. First, we will

\footnotetext{
${ }^{1}$ Fortunately, we have the analytical formula to compute FR. Thus, the update can be done extremely fast.
}

build the horizontal and vertical polar graphs [18], for the given floorplan denoted as $G_{H}$ and $G_{V}$, respectively. Let us take $G_{H}$ to illustrate how to build the horizontal polar graph. $G_{H}$ is a directed graph, each vertex $v$ in it corresponds to a vertical channel, and an edge $e=\left(v_{1}, v_{2}\right)$ corresponds to a circuit module whose left and right boundaries are adjacent to channels $v_{1}$ and $v_{2}$, respectively. For each vertex $v$, we assign its weight $w(v)$ to be its corresponding channel width. Similarly, for each edge $e$, we assign its weight $w(e)$ to be its corresponding module width. Graph $G_{V}$ can be built similarly. By running longest path algorithm on $G_{H} / G_{V}$, we can obtain the width/height of the chip (denoted by $W_{c} / H_{c}$ ). For those channels not on the critical paths in $G_{H} / G_{V}$, we will have some positive slacks in width/height, which lead to dead areas. It shall be noted that during buffer insertion, some circuit modules may have to shift to make room for buffer blocks (e.g., if no dead area exists). Therefore, a horizontal channel's height or a vertical channel's width may increase during BBP.

To better represent buffer block and facilitate easy data manipulation such as feasible region intersection, we divide each channel into a set of rectangular tiles. Then we compute for each tile $\tau$, its slack with respect to the longest path in the polar graph $G_{H}$ or $G_{V}$.

The while loop from lines 4 to 8 is the main part of our BBP algorithm. The iterative buffer insertion process will continue as long as there is still some net that needs buffer(s) to meet performance constraint. Each iteration of the while loop has two major steps: first, we will pick a best tile for buffer insertion (Pick_A_Tile); then, we will insert proper buffers into this tile (Insert_Buffers).

To pick the best tile in each iteration, the Pick_A_Tile routine works in the following two modes, depending on whether there exists some useful dead area for buffer insertion or not:

1. There exists some tile whose area slack is positive (due to dead area). In this case, buffers inserted into this tile will not increase the overall chip area as long as the total area of buffers inserted in the tile is smaller than this tile's area slack. For example of a tile $\tau$ in a vertical channel, suppose its width slack is $w_{\tau}$, and its height is $H_{\tau}$. Then we can insert as many as $\left\lfloor w_{\tau} H_{\tau} / A_{b}\right\rfloor$ buffers into tile $\tau$ without increasing the overall chip area, where $A_{b}$ is the area of a buffer. The actual number of buffers that can be inserted into $\tau$ may be smaller, since only those buffers whose FR intersects with tile $\tau$ can be candidates to be inserted into $\tau$. Therefore, the number of buffers that can be inserted into $\tau$, without chip area increase is $n_{\tau}=\min \left(\left\lfloor w_{\tau} H_{\tau} / A_{b}\right\rfloor, m_{\tau}\right)$, where $m_{\tau}$ is the number of buffers whose FR's intersect with tile $\tau$. Since we may have multiple tiles with positive slack (especially at the beginning of BBP), we will pick the one with largest $n_{\tau}$ because this greedy approach shall reduce the total number of buffer blocks, which is also our buffer block planning objective.

2. There is no tile with positive area slack. Then, any buffer insertion will increase the overall chip area. When some buffer is inserted into a tile, we have to shift some circuit modules. This shifting will make room for other tiles, so we will have some new positive-slack tiles. Our tile selection process will try to maximize such opportunity. Notice that as a buffer is inserted in $\tau$, other tiles in the same channel with $\tau$ will have positive area and tend to have buffers inserted in the future, thus the chance of buffers clustering increases. To maximize such effect, we will pick the channel that has the maximum buffer insertion demand and choose one tile in it. Note that in this scenario, since we need to expand the channel, we only insert one buffer into it to minimize the area increase.

Our strategy for Insert_Buffers into the tile $\tau$ that has just been picked by Pick_A_Tile also works in two modes, corresponding to those two in Pick_A_Tile: 
1. The tile $\tau$ has dead area. From case 1 in Pick_A_Tile, we know that $n_{\tau}$ buffers can be inserted into the tile. Meanwhile, there are $m_{\tau}$ buffer candidates whose FR's intersect with tile $\tau$, with $m_{\tau} \geq n_{\tau}$. Then if $n_{\tau}=m_{\tau}$, we will insert all these $m_{\tau}$ buffers; if $m_{\tau}>n_{\tau}$, we will only insert first $n_{\tau}$ buffers out of these $m_{\tau}$ buffers, sorted according to the increasing size of their FRs. Different from previous approaches that just inserts one buffer for one net, our approach inserts as many as $n_{\tau}$ buffers for $n_{\tau}$ different nets simultaneously. Since all of them are clustered into tile $\tau$, they form a natural buffer block.

2. The tile does not have dead area, but needs expansion to make room for any buffer insertion. In this case, we only insert one buffer, i.e., $n_{\tau}=1$. Again, if there are multiple buffers that can be inserted in this tile, we insert the one with tightest FR constraint.

After deciding how many and which $n_{\tau}$ buffers are inserted ("committed") into tile $\tau$, we will update the following information: (i) The feasible regions of "uncommitted" buffers in the same net for which we just inserted a buffer into $\tau$; (ii) The corresponding vertex (i.e., channel) weights in $G_{H}$ and/or $G_{V}$ that are affected by the insertion of the buffer block; (iii) The new chip dimension $W_{c}, H_{c}$ and the slacks for each channel and tile. Then we repeat the buffer insertion/clustering process until all buffers are placed. It shall be pointed that our BBP algorithm can handle both slicing and non-slicing floorplanning structures.

\section{Experimental Results}

We have implemented our buffer block planning algorithms using $\mathrm{C}++$ on an Intel Pentium-II machine with 256M-byte main memory. This section presents the experimental results. The parameters (refer to Table 1) used in our experiments are based on a $0.18 \mu \mathrm{m}$ technology in the NTRS'97 roadmap [16].

We have tested our algorithms on 11 circuits, as summarized in Table 2. The first six circuits are from MCNC benchmark [19], and the other five are randomly generated. In this paper, we focus on 2-pin nets, so we decompose each multi-pin net into a set of source-to-sink 2-pin nets ${ }^{2}$. We then compute the critical length for buffer insertion (defined to be the minimal interconnect length that buffer insertion is needed for delay reduction) using the analytical formula in [17]. We will use it to filter out short interconnects, i.e., if a net is shorter than the critical length, we will ignore it during buffer block planning since buffer insertion can not help reduce its delay. The initial floorplan for each circuit is generated by running the simulated tempering (an improved Monte Carlo technique of simulated annealing) algorithm as in [20]. For each net, we first compute its best delay by optimal buffer insertion $T_{\text {opt }}$ [17], and then randomly assign its delay budget to be $1.05 \sim 1.20 T_{\text {opt }}$.

We compare our BBP algorithm with a conventional buffer insertion algorithm without trying to plan buffer positions, i.e., at each iteration, a buffer is randomly picked and assigned to a feasible location, denoted as RDM algorithm. We run BBP and RDM under two different scenarios: one is RES where a buffer can only be located in its delay-minimal restricted position(s) (see Figure 3); and the other is FR where a buffer may be inserted anywhere in its feasible region. The results for the four different algorithmatic combinations are summarized in Table 3, where BBP/RES means BBP algorithm applied to scenario RES, RDM/FR means RDM applied to scenario FR, and so on.

In Table 3, we report for each algorithm combination: (i) the total number of buffers inserted to meet performance constraints

\footnotetext{
${ }^{2}$ Note that the number of 2-pin nets is possibly smaller than that of original nets (playout) because the power/ground and single-pin nets are excluded.
}

Table 2: Test circuit statistics.

\begin{tabular}{||r|r|r|r|r||}
\hline \hline circuit & \#modules & \# nets & \# pads & \#2-pin nets \\
\hline apte & 9 & 97 & 73 & 172 \\
\hline xerox & 10 & 203 & 2 & 455 \\
\hline hp & 11 & 83 & 45 & 226 \\
\hline ami33 & 33 & 123 & 43 & 363 \\
\hline ami49 & 49 & 408 & 22 & 545 \\
\hline playout & 62 & 2506 & 192 & 2150 \\
\hline ac3 & 27 & 212 & 75 & 446 \\
\hline$x c 5$ & 50 & 1005 & 2 & 2275 \\
\hline hc7 & 77 & 449 & 51 & 1450 \\
\hline$a 9 c 3$ & 147 & 1202 & 22 & 1613 \\
\hline$p c 2$ & 124 & 3126 & 192 & 4204 \\
\hline \hline
\end{tabular}

(buffers), (ii) the number of buffer blocks ( $\# B B$ ), (iii) the number of 2-pin nets that can meet their delay constraints (\#meet $)^{3}$, (iv) the chip area increase due to buffer insertion in percentage (area), and (v) the CPU time in second (cpu). It is interesting to observe from the table that

- Under the same RES scenario (i.e., only the restricted positions are allowed for buffer insertion), the RDM and BBP algorithms will have about the same number of buffers inserted and the same number of nets meeting their delay constraints. However, our BBP algorithm is able to explicitly cluster appropriate buffers together, so that it leads to significant area saving and much less number of buffer blocks than RDM algorithm. For example of circuit $p c 2$, the area increase of $\mathrm{BBP} / \mathrm{RES}$ is $3.21 \%$, whereas that of RDM/RES is about $9 \%$ (2.8x larger); the \#BB of BBP/RES is 542, whereas that of $\mathrm{RDM} / \mathrm{RES}$ is about 1290 (2.38x larger). The same conclusion about the comparison of BBP versus RDM holds for the FR scenario. It is also interesting to observe that BBP algorithm does not indeed increase CPU time from RDM. Actually, it may use slightly less run time. This is because during BBP, one buffer block (not just one buffer) can be determined at a time.

- Under the same algorithm, e.g., BBP, the usage of FR significantly increases the number of nets that can meet their delay constraints (for example of $a c 3$, from 289 to 369, a $28 \%$ increase). This is because our feasible region is usually much larger than the delay-minimal RES locations, so that one can avoid existing circuit/buffer blockages during buffer insertion. Note that as \#meet increases, the number of buffers inserted to meet performance constraints also increases accordingly from RES to FR. However, since the FR provides much more freedom during buffer clustering, the number of buffer blocks $(\# B B)$ in fact reduces (for example of of $a 9 c 3$, from 557 to 366 , a $34 \%$ reduction); and the area expansion due to buffer insertion is also less by using FR with better buffer clustering. Since the FR computation/update can be computed in constant time, the run times under FR scenario only increase slightly compared to those under RES. As a result, our largest example ( $p c 2$ with more than 13,000 buffers) only takes about $100 s$.

To summarize, it is obvious that the BBP/FR is the best combination among these four to meet delay targets, with very marginal

${ }^{3} \mathrm{~A}$ net will fail to meet its delay constraint if the given delay constraint is too tight, or its buffer's feasible region is fully occupied by existing circuit blocks. 
Table 3: Comparison of four different buffer insertion/planning algorithms.

\begin{tabular}{|c|c|c|c|c|c|c|c|c|c|c|}
\hline \multirow[b]{2}{*}{ circuit } & $\overline{\text { buffers }}$ & $\# B B$ & \#meet & $\overline{\text { area }}$ & $\overline{c \text { cpu(s) }}$ & buffers & $\# B B$ & \#meet & $\overline{\text { area }}$ & $\overline{c \text { cpu }(s)}$ \\
\hline & \multicolumn{5}{|c|}{ RDM/RES } & \multicolumn{5}{|c|}{ RDM/FR } \\
\hline apte & 157 & 74 & 81 & $1.50 \%$ & 0.14 & 181 & 93 & 98 & $1.81 \%$ & 0.25 \\
\hline xerox & 343 & 102 & 210 & $1.85 \%$ & 0.36 & 403 & 116 & 262 & $2.11 \%$ & 0.56 \\
\hline hp & 242 & 119 & 106 & $2.48 \%$ & 0.28 & 274 & 147 & 128 & $2.78 \%$ & 0.47 \\
\hline ami33 & 625 & 278 & 271 & $2.91 \%$ & 1.22 & 660 & 306 & 302 & $3.41 \%$ & 1.78 \\
\hline ami49 & 867 & 376 & 342 & $3.19 \%$ & 2.47 & 921 & 404 & 394 & $3.42 \%$ & 3.78 \\
\hline playout & 3997 & 581 & 1337 & $3.74 \%$ & 13.23 & 4244 & 730 & 1515 & $4.06 \%$ & 17.61 \\
\hline ac3 & 649 & 267 & 302 & $2.56 \%$ & 1.00 & 736 & 290 & 377 & $2.90 \%$ & 1.61 \\
\hline $\mathrm{xc5}$ & 2860 & 460 & 1379 & $4.20 \%$ & 7.95 & 3197 & 499 & 1727 & $4.21 \%$ & 11.83 \\
\hline hc7 & 2557 & 909 & 967 & $6.50 \%$ & 11.56 & 2662 & 941 & 1053 & $6.63 \%$ & 17.89 \\
\hline a9c3 & 4035 & 1082 & 1257 & $4.46 \%$ & 26.09 & 4236 & 1156 & 1416 & $4.66 \%$ & 34.80 \\
\hline \multirow[t]{2}{*}{$\mathrm{pc} 2$} & 12237 & 1290 & 2687 & $8.95 \%$ & 80.11 & 13157 & 1371 & 3339 & $8.99 \%$ & 122.34 \\
\hline & \multicolumn{5}{|c|}{ BBP/RES } & \multicolumn{5}{|c|}{$\overline{\mathrm{BBP} / \mathrm{FR}}$} \\
\hline apte & 168 & 48 & 90 & $0.80 \%$ & 0.14 & 185 & 34 & 102 & $0.69 \%$ & 0.23 \\
\hline xerox & 330 & 68 & 195 & $1.06 \%$ & 0.33 & 399 & 66 & 260 & $1.38 \%$ & 0.53 \\
\hline hp & 245 & 77 & 109 & $1.49 \%$ & 0.30 & 280 & 64 & 131 & $1.24 \%$ & 0.48 \\
\hline ami33 & 616 & 161 & 259 & $1.49 \%$ & 1.06 & 667 & 125 & 305 & $1.36 \%$ & 1.63 \\
\hline ami49 & 882 & 197 & 355 & $1.25 \%$ & 2.08 & 946 & 136 & 412 & $0.78 \%$ & 3.25 \\
\hline playout & 4016 & 245 & 1350 & $1.13 \%$ & 10.02 & 4263 & 201 & 1533 & $0.84 \%$ & 13.98 \\
\hline ac3 & 639 & 145 & 289 & $1.28 \%$ & 0.86 & 733 & 118 & 369 & $1.11 \%$ & 1.39 \\
\hline $\mathrm{xc5}$ & 2920 & 285 & 1431 & $2.58 \%$ & 6.38 & 3210 & 193 & 1739 & $1.79 \%$ & 10.16 \\
\hline hc7 & 2542 & 455 & 946 & $2.86 \%$ & 9.89 & 2693 & 299 & 1068 & $1.92 \%$ & 15.88 \\
\hline a9c3 & 4082 & 557 & 1293 & $1.83 \%$ & 21.13 & 4265 & 366 & 1446 & $0.89 \%$ & 29.20 \\
\hline $\mathrm{pc} 2$ & 12530 & 542 & 2837 & $3.21 \%$ & 63.91 & 13238 & 416 & 3462 & $2.02 \%$ & 99.84 \\
\hline
\end{tabular}

area increase (less than $2.1 \%$ for all test cases), least number of buffer blocks and comparable CPU times. It shall be noticed, however, that even under this best algorithm, there may still exist quite some nets that cannot meet their delay constraints under some given floorplan and timing budget. Therefore, it is important to have an interconnect-driven floorplanning engine to work closely with our $\mathrm{BBP} / \mathrm{FR}$ algorithm. We are currently working on it.

As an example, we show in Figure 5 the circuit buffer block layouts from RDM/RES and BBP/FR on circuit xerox.

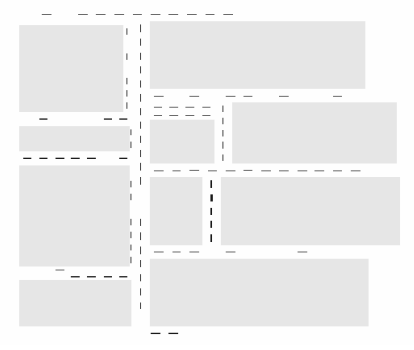

(a)

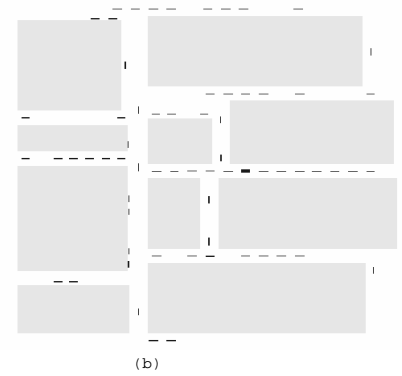

Figure 5: Floorplan and buffer block layouts of the MCNC circuit xerox by (a) RDM/RES; (b) BBP/FR. The ten big blocks are circuit functional modules, and the rest are buffer blocks.

\section{Conclusion}

In this paper, we introduced the concept of feasible region for buffer insertion and derive the analytical formula to compute FR under given delay constraint. We then proposed an effective buffer block planning (BBP) algorithm to automatically generate buffer blocks for interconnect optimization with chip area and buffer block number minimization. Experimental results showed that our BBP/FR algorithm leads to significant improvement over previous buffer insertion/planning algorithms.

Our buffer block planning bridges the gap between interconnect layout optimization and physical design. By constructing appropriate buffer blocks and determining the rough location of each buffer for every net, we can obtain more accurate, on-line interconnect estimation/prediction for wire length, congestion as well as delay with appropriate buffer insertion considered. Since buffer insertion is a key technique to reduce interconnect delay, we believe that effective buffer block planning will play a very important role in an interconnect-centric design flow for timing closure and design convergence.

\section{Acknowledgments}

The authors would like to thank Dr. Wilm Donath from IBM T. J. Watson Research Center, Dr. Norman Chang from HP Labs, and Dr. Lukas van Ginneken from Magma Design Automation for their helpful discussions.

\section{References}

[1] J. Cong, L. He, C.-K. Koh, and P. H. Madden, "Performance optimization of VLSI interconnect layout," Integration, the VLSI Journal, vol. 21, pp. 1-94, 1996.

[2] H. B. Bakoglu, Circuits, Interconnections, and Packaging for VLSI. Addison-Wesley, 1990.

[3] R. Otten, "Global wires harmful?," in Proc. Int. Symp. on Physical Design, pp. 104-109, Apr. 1998.

[4] J. Cong and D. Z. Pan, "Interconnect delay estimation models for synthesis and design planning," in Proc. Asia and South Pacific Design Automation Conf., pp. 97-100, Jan., 1999. 
[5] J. Cong, L. He, K.-Y. Khoo, C.-K. Koh, and D. Z. Pan, "Interconnect design for deep submicron ICs," in Proc. Int. Conf. on Computer Aided Design, pp. 478-485, 1997.

[6] J. Cong, "Challenges and opportunities for design innovations in nanometer technologies.," in SRC Working Papers, http://www.src.org/prg_mgmt/frontier.dgw, Dec. 1997.

[7] L. P. P. P. van Ginneken, "Buffer placement in distributed RCtree networks for minimal Elmore delay," in Proc. IEEE Int. Symp. on Circuits and Systems, pp. 865-868, 1990.

[8] J. Lillis, C. K. Cheng, and T. T. Y. Lin, "Optimal wire sizing and buffer insertion for low power and a generalized delay model," in Proc. Int. Conf. on Computer Aided Design, pp. 138-143, Nov. 1995.

[9] T. Okamoto and J. Cong, "Buffered Steiner tree construction with wire sizing for interconnect layout optimization," in Proc. Int. Conf. on Computer Aided Design, pp. 44-49, Nov. 1996.

[10] C. C. N. Chu and D. F. Wong, "Closed form solution to simultaneous buffer insertion/sizing and wire sizing," in Proc. Int. Symp. on Physical Design, pp. 192-197, 1997.

[11] W. M. Dai, B. Eschermann, E. S. Kuh, and M. Pedram, "Hierachical placement and floorplanning for bear," in IEEE Trans. on Computer-Aided Design, pp. 1335-1349, 1989.

[12] D. Wong and C. L. Liu, "Floorplan design of VLSI circuits," in Algorithmica, pp. 263-291, 1989.

[13] H. Murata, K. Fujiyoshi, S. Nakatake, and Y. Kajitani, "Rectangle-packing-based module placement," in Proc. of Int. Conf. on Computer-Aided Design, pp. 472-479, 1995.

[14] M. Kang, W. Dai, T. Dillinger, and D. LaPotin, "Delay bounded buffered tree construction for timing driven floorplanning," in Proc. of Int. Conf. on Computer-Aided Design, pp. 707-712, 1997.

[15] J. Cong, "An interconnect-centric design flow for nanometer technologies," in Proc. of Int'l Symp. on VLSI Technology, Systems, and Applications, pp. 54-57, June, 1999.

[16] Semiconductor Industry Association, National Technology Roadmap for Semiconductors, 1997.

[17] C. J. Alpert and A. Devgan, "Wire segmenting for improved buffer insertion," in Proc. Design Automation Conf., 1997.

[18] R. Otten, “Graphs in floor-plan design,” International Journal of Circuit Theory and Applications, vol. 16, pp. 391-410, Oct. 1988.

[19] "http://www.cbl.ncsu.edu/cbl_docs/lys92.html,"

[20] J. Cong, T. Kong, D. Xu, F. Liang, J. S. Liu, and W. H. Wong, "Relaxed simulated tempering for VLSI floorplan design," in Proc. Asia and South Pacific Design Automation Conf., pp. 13-16, Jan., 1999. 